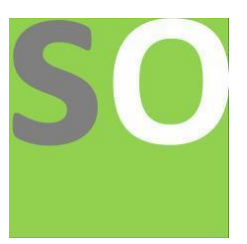

Article title: Upgrade social distancing into "Airflow Inaccessible Distancing" code validated by E. coli DH5a spray viable colony counting simulation and the technical validation of COVID-19 Airborne Infection UV Quarantine Devices Authors: Jingli Xing[1], Zongxin Wen[2], Yong Liu[3], Chao Li[4], Xuhui Chen[5], Chao Yang[6], Weiyi Liu[7], Qingzhong Guo[8], Dongwei Chen[9], Yi Yu Lai[10]

Affiliations: Innoen Gravitational Institute[1], Wuhan Torch Optoelectronic Technology Co., Ltd[2], McMaster University[3], Hubei Jucro Electric Co., Ltd.[4], Tsinghua University[5]

Orcid ids: 0000-0002-0113-8795[10]

Contact e-mail: yylai@innoen.org

License information: This work has been published open access under Creative Commons Attribution License http://creativecommons.org/licenses/by/4.0/, which permits unrestricted use, distribution, and reproduction in any medium, provided the original work is properly cited. Conditions, terms of use and publishing policy can be found at https://www.scienceopen.com/.

Preprint statement: This article is a preprint and has not been peer-reviewed, under consideration and submitted to ScienceOpen Preprints for open peer review.

Links to data: $\mathrm{https}: / /$ datadryad.org/stash

Funder: NA

DOI: 10.14293/S2199-1006.1.SOR-.PPADFAZ.v1

Preprint first posted online: 08 January 2021

Keywords: Airflow inaccessible distancing, UV quarantine, placebo infection rate 


\title{
Upgrade social distancing into “Airflow Inaccessible Distancing” code validated by E. coli DH5a spray viable colony counting simulation and the technical validation of COVID-19 Airborne Infection UV Quarantine® Devices
}

\author{
Jingli Xing ${ }^{1^{*}}$,Zongxin Wen ${ }^{2 *}$, Yong Liu ${ }^{3}$, Chao Li ${ }^{1}$, Xuhui Chen ${ }^{4}$, Chao Yang ${ }^{1}$, Weiyi Liu ${ }^{2}$, Qingzhong Guo ${ }^{2}$, Dongwei \\ $\mathrm{Chen}^{5}$, Yi Yu Lai ${ }^{1,5}$ \\ 1. Innoen® Gravitational Institute, Mississauga, L6W 1V1, ON, Canada \\ correspondence E-mail: yylai@innoen.org \\ 2. Hubei Jucro Electric Co., Ltd., Hubei 443200, China \\ 3.Wuhan Torch Optoelectronic Technology Co., Ltd, Wuhan 430074, China \\ 4. McMaster University, Hamilton, L8S 4L8, ON, Canada \\ 5. Medical Center, Medical Science Building B343, Tsinghua University, Beijing 100084, China \\ * These authors contributed equally to this work
}

\begin{abstract}
While global attentions focus on COVID-19 immunological solutions, we believe a future possible vaccine will unexceptionally compromise to immunosenescence which will leave those older than 55 years of age inactive to any immobilization. We then validate the UV quarantine ${ }^{\circledR}$ system that shielding the UV hurt instead of segregating the contagious agents in an environment validated by $10^{6}$ cell $/ \mathrm{ml} \mathrm{E.coli}$ DH5 $\alpha$ spray viable colony simulation. Various experimental designs reveal that the social distancing rule should be upgraded to the "Airflow Inaccessible Distancing" code due to the undetectable small-sized infectious aerosols, saturation secondary infection from various filtering devices \& quarantine spaces, and the mixing efficiency of conventional chemical sanitizers. The new code and its integrated COVID-19 Airborne Infection UV quarantine ${ }^{\circledR}$ Devices are globally the highest-efficiency systems for stopping COVID-19 from spreading under general social conditions. Portable UVC 253.7nm meters can release the safety concerns for public self-made or commercially available devices. Meanwhile, an acrylic system and relevant methods are developed to validate the performance of UV quarantine $®$ devices and conventional masks under real applications. Also, a $33 \%$ placebo infection rate is suggested as the minimum requirement for Phase 3 clinical trials of all medical devices, vaccines, or drugs intended for COVID-19 due to the existence of human respiratory rhyme.
\end{abstract}

\section{Introduction}

The SARS-CoV-2 virus is continuing its spread across the world, with more than 10 million confirmed cases in 212 countries; over 500,000 deaths worldwide by 30 June 2020[1]. In the coronavirus crisis, 
people may persuade for immunological solutions. However, to date, all the available technologies or theories, still limited to the range of acquired immunity (antigen-specific immunity). Little is known or capable of handling the root cause level innate immunity (non-specific immune protection). The success rate of a vaccine is inversely proportional to its required innate immunity since this requirement makes the immunization effectiveness into strongly age-dependence. Cancer is an extreme example that $100 \%$ depends on innate immunity since no external antigen immunized. Therefore, design a vaccine against tumor cells will be methodologically inappropriate; young people don't need it or unknown of the competence immunization stage, for older people who close to the cancerous symptom stage, it becomes useless since innate immunity has already substantially decayed. For other vaccine intended illnesses, with the decreasing of required innate immunity, they will gradually adapt to the method. Ideal targets for immunization should be those problems that require less or zero innate immunity potential, with a sensitive acquired immune response, and also fewer mutation varieties. Innate immunity is strongly age-related, inevitably results in immunosenescence [2], which will leave those older than 55 years can't be activated for immune protection by any immunization technology. Immunosenescence is also the fundamental restriction that it is impossible to manipulate any immunization indicators, products, or technologies to improve the human lifespan. (Even averagely to increase 3 weeks of lifespan at a population level (>1 million people) is so challenging.)

For the case of COVID-19, it is still unknown how much innate immunity potential and how much adaptive immune response sensitivity is required for a successful COVID-19 infection prevention or even curing vaccine to present, also unknown of the mutation rates of the malignant virus types. However, from the fact that the envelope of the virus possesses human cell membrane ingredients that detached from the host, we can roughly believe that the protein \& RNA particle can be dealt with adaptive immunity, and the enveloping part must rely on innate immunity to counteract, then we can understand the real technological challenging for an effective vaccine to existing. Even if a vaccine against SARS-CoV-2 can work out in the future, a physical control method, if available, should be prioritized rather than that of a vaccine. For example, HIV can be prevented in various physical ways at a higher successful rate; thus, there is less need for a vaccine. Even if a vaccine against HIV is available in a cost-effective way, it can only serve as compensation for people in case of neglect while using physical isolation. It is groundless to require people to use a vaccine to replace the available physical quarantine, provide in reality a vaccine that can fully replace the latex film is still notoriously challenging. Now, the COVID-19 case is similar; the physical quarantine should be placed in a priority position. It must be a general principle of our human society partially due to the technological difficulty in manipulating innate immunity, and also due to a possible successful vaccine is highly likely to make substantial numbers of immunized persons into asymptomatic coronavirus carriers that are still capable of transmitting the lethal virus to others. Moreover, the complication limit of a vaccine is still one of the reasons. (As is known, there are substantial complications[3-13] from COVID-19 infected people. Even if a future vaccine can efficiently remove all the SARS-CoV-2 virus from a patient under the most ideal condition, it can't remove all the complications, patients still need innate immunity potential to recover from the complications, this phenomenon can be defined as the complication limit of a vaccine. Due to this limit, a vaccine will not fit for already infected and complication obvious patients, especially innate immunity decaying people.) From a social point of view, we should realize that aggressive infections such as the COVID-19 are not a problem that only impacts the vaccine user but will also significantly infects people nearby this vaccine user. For this reason, all future possible vaccines should possess immunization failure financial insurance compensation with age classification before finally going to the market, even if they have statistically passed three phases of clinical validation with a $50 \%$ success rate of stopping the infection under current standards. 
For the less attention physical quarantine method, a website has published the UV quarantine method on February 05, 2020. Also, a journal article [14] has put on top of it since March 05, 2020. By convention, quarantine the contagious agents is the fundamental way to stop infection; masks, protective clothes, chemical sanitation, and quarantine hospitals compose of the system elements. Different from this convention, the UV quarantine system [14] creates a partial or fully UV disinfecting working environment; people on-site use some UV protections to avoid radiation from attaining their naked skin. Three categories of UV protections in the paper are UV radiation box, UV radiation wall, and UV hood (now the term UV hood has been registered as "infection UV quarantine device"); they are self-made from some simple materials or purchased commercial replacements, quite convenient for unprofessional public usage. UV quarantine procedure [14] in the paper composed of a 30 min UV disinfection for utensils, equipment, or spaces, etc., in the family or public regions, then switch on UV radiation boxes (walls) if more than one people need to share them. In severely infected regions or high frequency visited areas, Airborne Infection UV Quarantine Devices will replace the UV radiation boxes (walls).

To experimentally validate the UV quarantine system, we need to review the critical control point of the elements of the conventional quarantine system - masks, helmets, and protective clothes. As in (Fig. 1a), the area marked with red color can be defined as "naked skin contact margin", the equivalent risk regions can be found in the helmet designs for preventing COVID-19 infection in (Fig. 1b). As is known, viruses are too smaller than bacteria to be "filter" by something, bacterial filters that are designed for particle sizes not less than $0.2 \mu \mathrm{m}$ need vacuuming plus a long period to remove targets. Therefore, even if we only consider bacteria in aerosols or droplets, filter mechanisms can't satisfy the human respiration volume requirements. (For those who never use bacterial filters, a simple experiment can alternatively be done. Just wearing a mask and use something like harmless glue or a stick to seal all the "naked skin contact margin" marked in (Fig. 1a), not allow any air leak from these regions, we can soon find it becomes reluctant to breathe. For "famous" masks like N95, this way even enough to suffocate the users due to the lower filtering efficiency. This simple experiment reveals that most of the airflow has never passed the functional filtering parts of conventional masks, only leaking out from the "naked skin contact margin".) Effective gas masks as in (Fig. 1c) must rely on two prerequisite designs:

1) Strictly design to avoid the virus invading from the "naked skin contact margin". (Industrial gas mask design requires airtight validation with less than $1 \%$ air leaking while fixed on a human face. In contrast, most conventional masks including N95 will leak out over 70\% of the airflow from their functional filtering parts.)

2) Take an active carbon cylinder or similar device to absorption the viruses. It is an absorption process instead of a filter; therefore, it can satisfy human respiration volume requirements as well as removing the pollutants. For those kinds of designs, active carbon cylinders must be renewed in a certain period after saturation. (The renewing period for most commercial cylinder products is lower than 200 hours.) Up till now, no technology can feasibly replace active carbon absorption devices.

From the above two technological prerequisite conditions we could know, the weaknesses of the elements of conventional quarantine systems, such as masks or helmets (without active carbon cylinder design), are still due to the naked skin contact margin leaking and the restricted exchange of air volume. Protective clothes must connect with advanced gas masks to avoid naked skin contact margin leaking; otherwise, they will easily lose their protective functions. Also, quarantine hospitals will fall into saturation secondary infection curves [15] like various conventional masks, which means those who use saturated 
masks or hospitals, will inevitably inhale more viruses than those who not using them. They have become secondary infectious sources after certain thresholds. The chemical sanitation method can only deal with hard surface decontamination, quite difficult for aerosol disinfection in the airflow due to small-sized particle mixing efficiency. We can understand that substantial global COVID-19 casualties come from the weakness of the conventional quarantine system, especially the "naked skin contact margin" leaking from various masks. (85\% of the global infected people till today have already worn diverse masks.)

Figure 1 | The weakness of the conventional quarantine system and challenging of chemical sanitation system
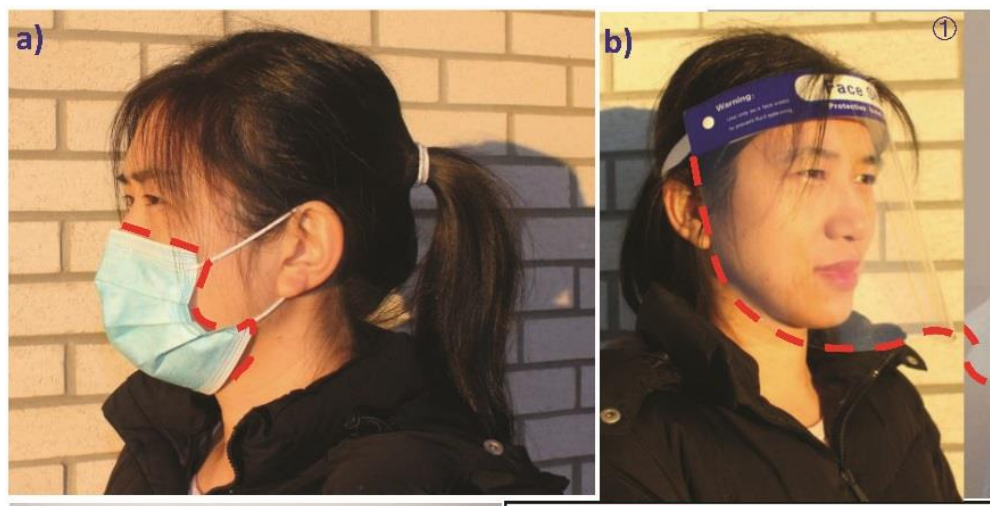

c)
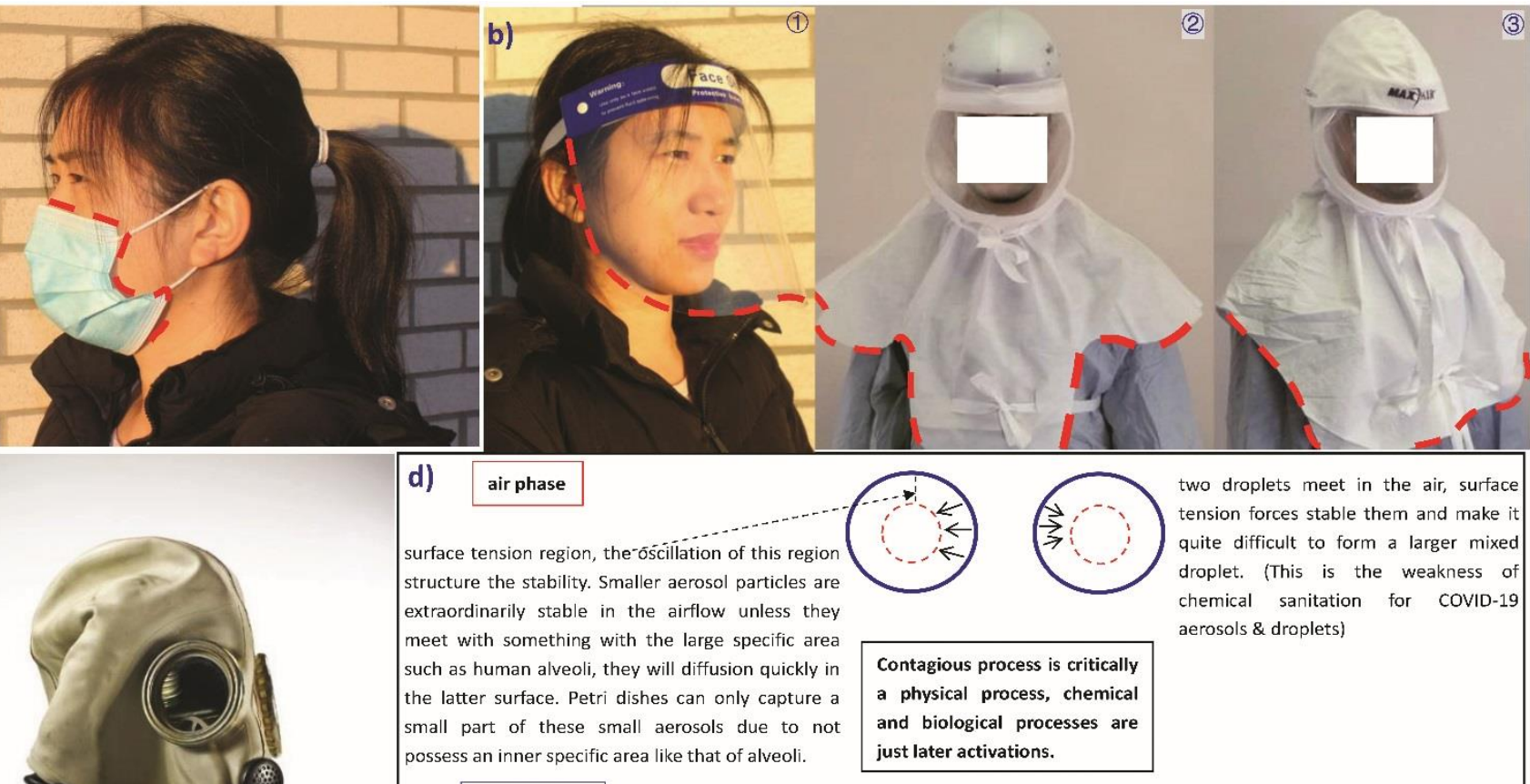

c)

two droplets meet in the air, surface tension forces stable them and make it quite difficult to form a larger mixed droplet. (This is the weakness of chemical sanitation for COVID-19 aerosols \& droplets)

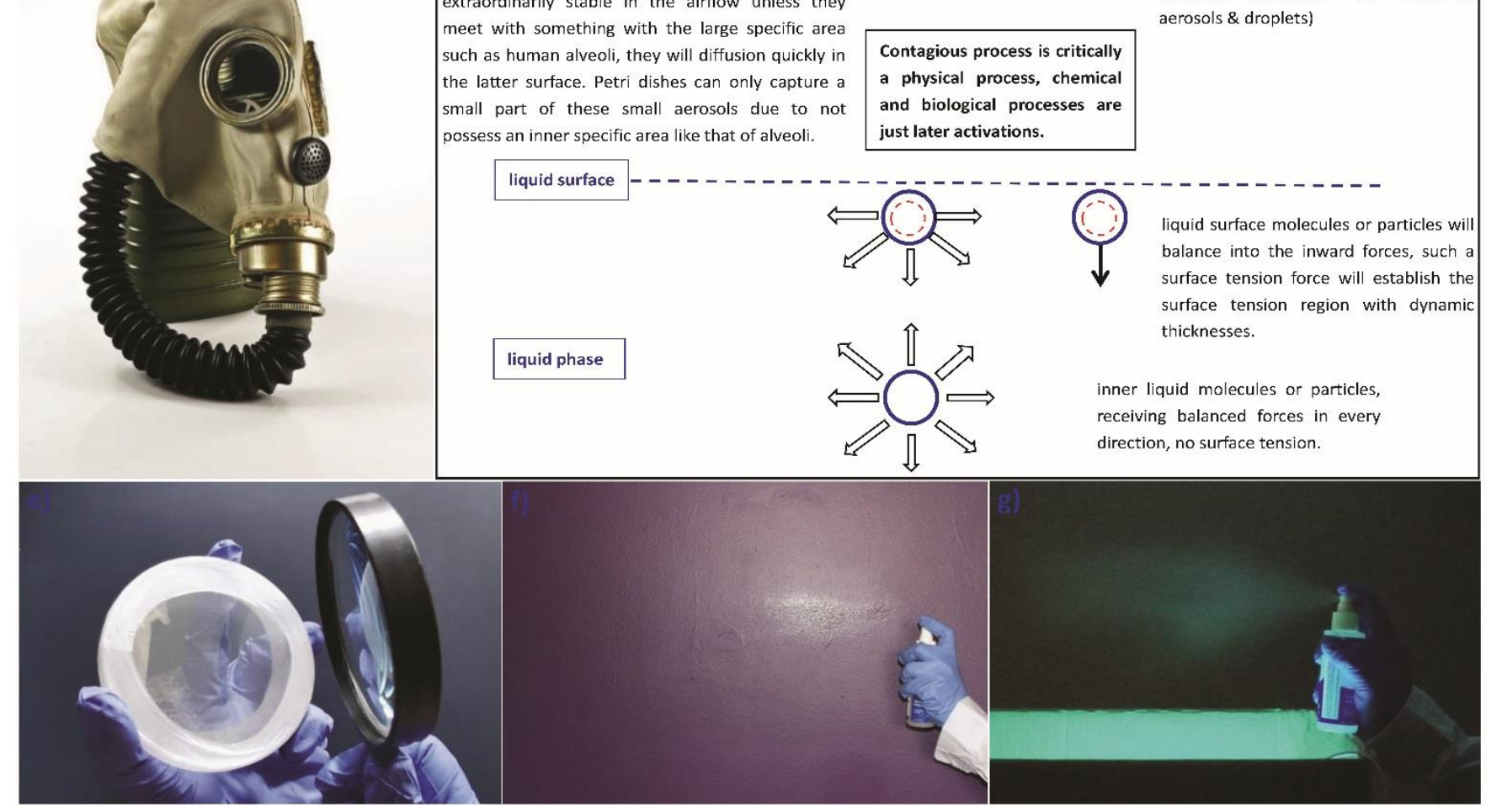

a) naked skin contact margin of a common mask (mark with red color). b) equivalent naked skin contact margins of some of the helmet designs, coronavirus invading will emerge from there with the increasing of staying time of infected human respiratory air. c) an advanced gas mask with an active carbon cylinder or similar design. Most gas mask products on the market are designed to follow 200-hour-functional 
life standard, which means every 200 hours a user must renew the active carbon cylinder. d) the weakness of chemical sanitation for small aerosol \& droplet particles in airflow due to surface tension and some other mechanisms, this challenge includes liquid particles with some solid components to reduce the surface tension. e) after preparing, each agar plate needs to be sealed with a parafilm, incubate at $37^{\circ} \mathrm{C}$ for 24 hours; discarded those with colony growth, and store with the original seal in a refrigerator $\left(0-4^{\circ} \mathrm{C}\right)$. While use, the sealed plates need to balance the temperature, and after capturing, plates also need to be sealed by a parafilm. While observing, open the cap and use a 5$10 \times$ magnifying glass. f) the spraying bottle with $10^{6} \mathrm{DH} 5 \alpha$ suspension leaves liquid marks on the wall. (The length of this mark is less than $40 \mathrm{~cm}) . \mathrm{g}$ ) the spraying bottle with tip $20 \mathrm{~cm}$ above a self-made UV radiation box loaded with DH5 $\alpha$ suspension for spraying. We can visually watch the spraying smog from the $10^{6}$ cells $/ \mathrm{ml}$ DH5 $\alpha$ sprays over the UV radiation inside the box at a dark background reflecting by the shining. (This method can maximize the image of visual spraying smog, of which the visual distance of the spraying smog can reach $60 \mathrm{~cm}$ in this experiment. This $60 \mathrm{~cm}$ distance should be the maximum distance in which most large droplets from the spraying bottle can reach.) From here we can see, the spraying smog only reaches a small range, while invisible small-sized aerosol particles can reach and viable in a remote distance up to 3 -meter. This UV radiation box is made from a cardboard box with $54 \times 33 \times 44 \mathrm{~cm}$ with a thickness of $4.5 \mathrm{~mm}$. A commercial UV meter with a resolution of $0.1 \mu \mathrm{w} / \mathrm{m}^{2}$ can't detect any UV leaking by directly attaching the sensor on the whole outside wall of the box while it shines UV germicidal lamps up to 60W inside. In all experiments with the UV radiation boxes (walls), we use a $30 \mathrm{~W}$ UV germicidal lamp inside. (Supplementary Movie 1) shows the E.coli suspension spray over the radiation box.

UV sterilization as technology has experienced a long history of over 100 years. Early as 1878, Arthur Downes and Thomas P. Blunt published a paper describing the sterilization of bacteria [16]. And the 1903 Nobel Prize for Medicine was awarded to Niels Finsen for his use of UV against lupus vulgaris, tuberculosis of the skin [17]. UV disinfection is used in a variety of applications, such as food, air, and water purification. According to current evidence, the COVID-19 virus is primarily transmitted between people through respiratory aerosols $(<10-\mu \mathrm{m})$, droplets $(>10-\mu \mathrm{m})$, and contact routes [18-22]. Airborne droplets can persist in the air for several minutes, and the smaller aerosols do not rapidly settle and can persist for longer durations to hours [19]. The SARS-CoV-2 virus has been found to remain viable in aerosols for $3 \mathrm{~h}$, while it, in the form of droplets, is more stable on plastic and stainless steel, copper, cardboard, and glass with durations detected up to $72,4,24$, and $84 \mathrm{~h}$, respectively[20]. The social distancing rule of 2-meter is insufficient [21]. Ventilation airflow complicates the infection routes [22]. UV disinfection fits for transparent media; therefore, it is feasible to use UV to disinfect the airborne COVID-19 aerosols \& droplets. Also, we must totally disregard the chemical sanitation for airborne microbes. The prerequisite condition for chemical sanitation is the mixture rate. If the airborne coronaviruses reside inside aerosols less than 1- $\mu \mathrm{m}$ and we want to use chemicals to kill them. Then the chemical solution must form less than $1-\mu \mathrm{m}$ aerosol particles or at least can establish something to mix well with these less than 1- $\mu$ m COVID-19 aerosol particles. In real environmental conditions, there exists complex electrostatic repulsion and surface tension among airborne liquid particles which size less than $1-\mu \mathrm{m}$, they reside in the air for months and still not condense or precipitate. It is noted that the size of aerosol generated by speaking and normal breathing is similar, ranging from 0.75 to $1.1-\mu \mathrm{m}$, quite smaller than those generated by coughing or sneezing, i.e., 5- $\mu \mathrm{m}$ [19]. This means the aerosols generated by speaking and normal breathing can stay longer, far-reaching, and spreading fast in the airflow. All these facts make chemical sanitation of SARS-CoV-2 viruses become notorious challenging even for professional people with advanced atomization spraying equipment. People just macrocosmically see the chemical smog they sprayed full of room space or open air; however, the COVID-19 aerosol particles still microcosmically untouched with chemical liquid particles, surface tension or some complex reasons offer them a higher percentage of "elastic collision" to avoid mixing, then after the chemical dissipated and people begin to use the room, the viruses inside the small aerosol particles emerge again. We can understand that a higher percentage of global COVID-19 casualties come from incorrectly rely on chemical sanitation for airborne viruses in a room that has been visited or used by heavily infected people, this way only fits for hard surface sanitation, almost useless for small aerosol in the airflow. It is regretted 
that almost all global hospitals largely rely on chemical sanitation; therefore, inevitably become the secondary sources of infection or viral assembly sites instead of a place that capable of cleaning the virus. (All hospitals only equip UV lamps in surgical rooms; other regions still rely on chemical sanitation.) Contrary to chemical solutions, UV disinfection targets follow the smaller size of the viral aerosols \& droplets, the more effective, show great advantages over chemical sanitation. In Petri dish experiments, UVC can eradicate bacteria in seconds [23] as below:

Table 1 | Direct UVC exposure time required to achieve eradication (0\% growth) [23]

\begin{tabular}{cc} 
Organism & $\begin{array}{c}\text { UVC exposure duration } \\
\text { (seconds) }\end{array}$ \\
\hline Methicillin-susceptible Staphylococcus aureus (MSSA) & 15 \\
Methicillin-resistant Staphylococcus aureus (MRSA) & 10 \\
Methicillin-susceptible, coagulase-negative Staphylococcus (MSCONS) & 10 \\
Methicillin-resistant, coagulase negative Staphylococcus (MRCONS) & 5 \\
Streptococcus pyogenes & 5 \\
Enterococcus species & 15 \\
\hline
\end{tabular}

For airborne status that different from the Petri dish conditions, there is no clear data for the UVC bacterial sterilization since people never realize that there are significant differences between the germicidal curves on a hard surface and inside airflow for chemical sanitation, and also due to the difficulty in the sampling of small-sized aerosols for physical parameter study. For COVID-19, the eradiation time should be significantly less than those of bacteria under an airborne state. This makes UV becomes the only irreplaceable and imperative method for airborne microbes. Due to the difficulty of culturing and the risk of handling coronavirus specimens, we use E. coli DH5 $\alpha$ suspension sprays to simulate the coronavirus transmission and to validate the social distancing. This low-risk species has been widely used as a bio contaminant indicator for food, water, and air for over one century. The industry standard has been calibrated to base on the notion that the increase of E. coli quantity in a sample means the increase of certain targeted bio contaminant(s), and the decrease also means the decrease of the targeted bio contaminant(s). Now, the quiddity of using viable E. coli colony counting to calibrate the aerosol contagious COVID-19 viral concentration still follows this canonical standard. Such a simulation should be reliable as the guidance for stopping in vitro COVID-19 infection, albeit it can't be used for human in vivo COVID-19 mobility patterns inside the body. The results of the simulation equivalent to those acquired from other methods and reliably validate the new "Airflow Inaccessible Distancing" code, also validate the COVID-19 Airborne Infection UV Quarantine ${ }^{\circledR}$ Devices, or commonly called UV masks that derived from this code.

\section{Results}

1. Validation of the $30 \mathrm{~min}$ UV radiation pre-disinfection protocol for airborne microbes

The 30min UV radiation pre-disinfection protocol [14] for public regions and family rooms originated from biosafety cabinet and industrial cleanroom procedures which have been applied in research labs and pharmaceutical industrials for quite a long time. Transfer this protocol to public regions not only due to the powerful virus eradication effects but also due to there is no other choice available for spaces that have 
been used by a patient, either symptomatic or asymptomatic coronavirus carriers. The weakness of chemical sanitation for small-sized virial aerosol particles due to the mixture efficiency has been revealed in (Fig. 1d). The only way to disinfect these regions becomes UV radiation and is also easy to use for the non-professional public. Now we will validate this protocol:

In an ordinary family environment, in the middle of the chosen room, locate a 1-meter height center, a 2meter, and a 3-meter circle on the same height plane. On each circle choose evenly distributed 5 sampling points, all these sampling points are on a platform $1 \mathrm{~m}$ height from the ground and $1.5 \mathrm{~m}$ lower from the 40W UV lamps in the middle. (All the sampling points need to away from the ground, ceiling, wall, door, window, ventilation inlets or outlets, etc. for at least 1 meter. Above 1-meter perpendicular of each sampling point, there is an $8 \mathrm{~W}$ UV lamp, a total of 10 sets of $8 \mathrm{~W}$ UV lamps on 10 sampling points, all are double-capped quartz glass UV germicidal lamps within first 100 hours of operation. In all the experiments, the ventilation system is shut off. The operator uses sterilized protective cloth, hairnet, gloves, shoe covers, and try to avoid making extra air turbulence.)

Within 1 hour after 30 min of UV radiation, open the sealed plates (as prepared in (Fig. 1e)) with LB (Luria-Bertani) agar on each point and expose for $5 \mathrm{~min} \& 15 \mathrm{~min}$ duration at a different time of 20min, $40 \mathrm{~min}, 60 \mathrm{~min}$ after the endpoint of the protocol, incubate plates on $37^{\circ} \mathrm{C}$ for 48 hours, check the colony counting with 5-10 times magnifying glass (Fig.1e). (If two or more overlapped colonies can be discerned, then counting as the discerned colony number.) CKs need the room hasn't used any UV lamps in a week, start from the same time point (such as $10 \mathrm{am}$ ) with the UV protocol treatment.

Table 2 | Static validation of the $30 \mathrm{~min}$ UV radiation protocol in an ordinary family room

\begin{tabular}{|c|c|c|c|c|c|c|c|c|c|c|}
\hline \multirow{2}{*}{$\begin{array}{l}\text { CKs: } \min \text { after a point, } \\
\text { no UV protocol* } \\
(\min )\end{array}$} & \multirow{2}{*}{$\begin{array}{c}\text { capturing } \\
\text { time } \\
(\min )\end{array}$} & \multicolumn{7}{|c|}{ colony counting (colony/plate) } & \multirow[b]{2}{*}{ Ave } & \multirow[b]{2}{*}{ STD } \\
\hline & & 0 & Ave & STD & $2 \mathrm{~m}$ & Ave & STD & $3 \mathrm{~m}$ & & \\
\hline \multirow[t]{2}{*}{20} & 5 & $17,15,22,13,20$ & 17.4 & 3.65 & $15,21,17,16,20$ & 17.8 & 2.59 & $19,15,15,13,14$ & 15.2 & 2.28 \\
\hline & 15 & $20,21,18,22,19$ & 20.0 & 1.58 & $25,23,18,22,19$ & 21.4 & 2.88 & $21,26,18,22,19$ & 21.2 & 3.11 \\
\hline \multirow[t]{2}{*}{40} & 5 & $15,14,13,19,21$ & 16.4 & 3.44 & $16,15,18,23,20$ & 18.4 & 3.21 & $17,15,20,18,20$ & 18.0 & 2.12 \\
\hline & 15 & $18,21,18,22,15$ & 18.8 & 2.77 & $20,21,18,22,24$ & 21.2 & 2.59 & $24,25,18,22,19$ & 21.6 & 3.05 \\
\hline \multirow[t]{2}{*}{60} & 5 & $18,18,19,14,21$ & 18.0 & 2.55 & $22,15,14,19,20$ & 18.0 & 3.39 & $16,18,22,26,20$ & 20.4 & 3.85 \\
\hline & 15 & $16,20,25,22,21$ & 20.8 & 3.27 & $28,20,18,16,19$ & 20.2 & 4.60 & $22,26,18,22,21$ & 21.8 & 2.86 \\
\hline $\begin{array}{l}\text { Treatments: min after } \\
\text { UV protocol endpoint }\end{array}$ & $\begin{array}{l}\text { capturing } \\
\text { time }\end{array}$ & & & & & & & & & \\
\hline$(\min )$ & $(\min )$ & 0 & Ave & STD & $2 \mathrm{~m}$ & Ave & STD & $3 \mathrm{~m}$ & Ave & STD \\
\hline \multirow[t]{2}{*}{20} & 5 & $3,0,0,4,0$ & 1.4 & 1.95 & $0,0,5,1,3$ & 1.8 & 2.17 & $4,0,3,0,0$ & 1.4 & 1.95 \\
\hline & 15 & $0,5,4,0,2$ & 2.2 & 2.28 & $4,8,0,4,0$ & 3.2 & 3.35 & $2,0,0,1,0$ & 0.6 & 0.89 \\
\hline \multirow[t]{2}{*}{40} & 5 & $0,0,1,1,7$ & 1.8 & 2.95 & $3,5,0,2,1$ & 2.2 & 1.92 & $1,1,7,0,3$ & 2.4 & 2.79 \\
\hline & 15 & $0,2,5,6,9$ & 4.4 & 3.51 & $8,6,7,11,0$ & 6.4 & 4.04 & $3,2,7,9,0$ & 4.2 & 3.70 \\
\hline \multirow[t]{2}{*}{60} & 5 & $1,1,3,0,4$ & 1.8 & 1.64 & $5,1,0,6,0$ & 2.4 & 2.88 & $7,3,0,5,8$ & 4.6 & 3.21 \\
\hline & 15 & $2,4,0,3,5$ & 2.8 & 1.92 & $7,6,0,5,0$ & 3.6 & 3.36 & $3,9,3,4,0$ & 3.8 & 3.27 \\
\hline
\end{tabular}

From the results, no matter after 20, 40, 60 min of the endpoint of UV protocol, at $0 \mathrm{~m}, 2 \mathrm{~m}, 3 \mathrm{~m}$ from the center, the viable colony counting with UV protocol and without the UV protocol is significantly different. This means the 30 min UV disinfection protocol in a static ordinary room significantly kills most of the airborne microbes and the disinfection state can at least last for one hour after the protocol under a general condition. In real applications, effective UV germicidal lamps can kill $99 \%$ surface bacteria in seconds ${ }^{12}$, laboratory or industrial cleanroom procedures require $30 \mathrm{~min}$ disinfection only due to regulatory minimize 
the risk. The survival rate of the RNA coronaviruses will be quite lower than that of bacteria; therefore, they can also be eradicated in seconds. (Petri dish airborne viable microbe capturing technology has been applied in food \& pharmaceutical industries for near one century. Even the modern mainstream disciplines such as molecular biology still fail to find any replacement assay for this canonical application technology. We have no other choice but to rely on it for the simulation.) This simulation validates the effectiveness of UV disinfection for airborne microbes in a static room; however, not clearly reveal the UV's advantages over moving small-sized aerosol particles. Now we go to the dynamic spraying simulation, 5 min capturing time is chosen:

A commercial spray bottle is selected by checking that it can spray DH5 $\alpha$ exponential stage LB broth into visually fine droplets. DH5 $\alpha$ exponential stage LB broth suspension from $250 \mathrm{ml}$ flasks on a rotary shaker needs to pass an ordinary chemical filter paper first before adjusting by a Petroff-Hausser chamber to $10^{6}$ cell $/ \mathrm{ml}$ and then spraying, in each spray it drives out a $0.7-1.5 \mathrm{ml}$ suspension. (Fig.1f) shows the length of liquid mark on the wall make by this spraying bottle is less than $40 \mathrm{~cm}$. (Fig.1g) shows the visual smog made by this bottle over a UV radiation box under dark background can reach around $60 \mathrm{~cm}$. This $60 \mathrm{~cm}$ distance should be the maximum distance in which most large droplets from the spraying bottle can reach. For the invisible small-sized aerosol \& droplet particles, no literature clears about how long they can stay in the airflow, how far away they can travel, and the speed of moving. We then have to use an indirect method for evaluation.

Same with those in the static validation for room and sampling point designs, 30 min after the time endpoint of the UV protocol, three treatments in (Tab.3) with the same colony counting method in static room validation, one treatment need the spraying tip $20 \mathrm{~cm}$ above the top of the UV radiation box with a 30W UV lamp shinning inside while spraying horizontally point to each sampling point, as in (Fig.1g).

Table 3 | Dynamic spraying validation of $30 \mathrm{~min}$ UV protocol and the UV radiation from a $30 \mathrm{~W}$ UV germicidal lamp for moving airborne infectious agents at a $64 \mathrm{~cm}$ height of the perpendicular distance

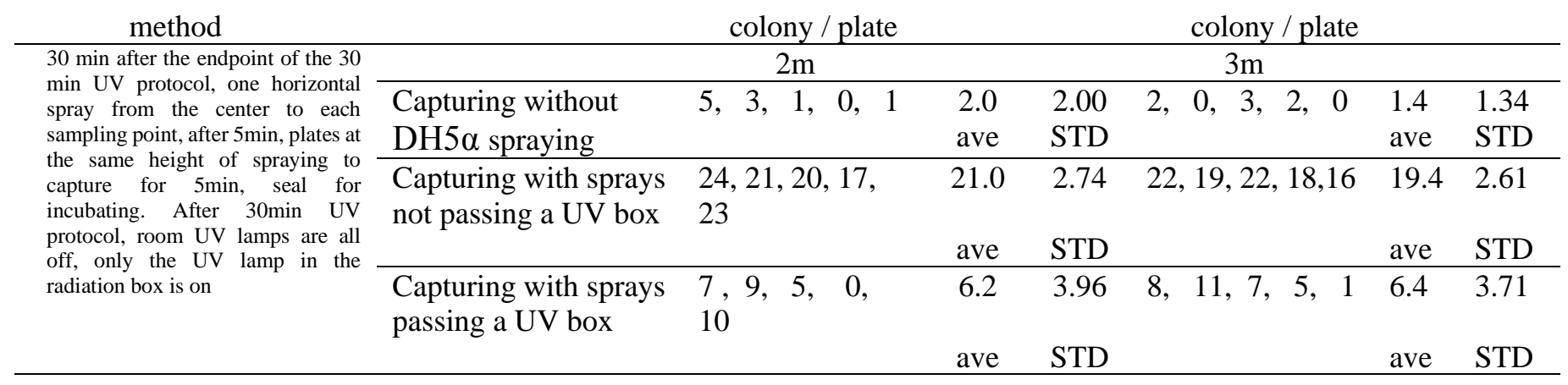

From the table, similar results with those in (Tab.2), at $2 \mathrm{~m}$ and $3 \mathrm{~m}, 30 \mathrm{~min}$ after the time endpoint of UV protocol, spraying treatment increases the average viable capturing colony from 2.0, 1.4, to 21.0, 19.4, respectively; STD also significantly increased. Albeit the liquid mark or smog produced by the spraying bottle can visibly only attain less than $60 \mathrm{~cm}$ distance, at the sampling point as far as $2 \mathrm{~m}$ and $3 \mathrm{~m}$, only allow $5 \mathrm{~min}$ of traveling time, colony counting significantly increased around to 10 folds. This is due to the attainment and viability of invisible small-sized aerosol \& droplet particles, which similar to those results described in other literature. (We lack larger indoor space for the experiment; it is estimated small- 
sized aerosol particles can travel much more than $3 \mathrm{~m}$ in most indoor spaces even without the driving effects of the ventilation system.)

For sprays passing the UV radiation box (Fig.1g), at $2 \mathrm{~m}$ and $3 \mathrm{~m}$ sampling point, average colony counting reduced from 21, 19.4 to 6.2, 6.4 respectively; this means UV radiation does can kill the airborne microbes in the small-sized aerosol particles of moving airflow. (It is difficult to know exactly the speed of these small-sized aerosol particles. Nearby the spraying tips, droplets moving very fast; however, over $60 \mathrm{~cm}$ how small-sized aerosol particles move to $2 \mathrm{~m}$ or $3 \mathrm{~m}$ and viable into plates is totally unclear. Therefore, it is difficult to estimate which kind of airflow velocity will increase or decrease the infectious agent concentrations. Our simulation can only indirectly show that these small-sized aerosol or droplet infectious particles do travel to viable the far away Petri dishes with a higher impact, and also shows UV radiation from the UV radiation box can significantly kill these moving infectious agents inside airflow under the experimental conditions. These experiments are performed for indoor space, for outdoor environments, windless public regions will similar to our indoor static simulation. Under breeze outdoor condition, the wind parameters as the factors will also add complexity to the process, airflow can drive off aerosol coronavirus in a region, it also can carry viruses to infect remote people under certain conditions. It is unreasonable to believe that the social distancing rule is safer for outdoor public regions than that of it for indoor public regions.) However, this result is enough to suggest the upgrading of the "social distancing" rule into the "Airflow Inaccessible Distancing" code. Also, this validation shows the UV radiation box (wall) is significantly effective for small aerosols.

2. Validation of COVID-19 Airborne Infection UV quarantine ${ }^{\circledR}$ Medical Device without device mounted UV germicidal lamps (Canadian Class II medical device with ID: 319987HB\#, 319987HD\#; can be self-made. The common name of which is UV hood.)

With the same spraying bottle prepared and applied as before, open all the UV lamps in the room and the experimental operator uses COVID-19 Airborne Infection UV quarantine ${ }^{\circledR}$ Devices plus some other assistant UV protections which safety has been validated; then from the center to spray 5 times with 1second interval to horizontally point to each sampling point, after $5 \mathrm{~min}$ of the endpoint of spraying, open sealed plates to capture $5 \mathrm{~min}$ for each point on $2 \mathrm{~m}$ and $3 \mathrm{~m}$, (for $0 \mathrm{~m}$ sampling point which represent the spraying bottle location, after 10s of the endpoint of finishing spraying to each sampling point, directly open 5 plates and each one only expose for the 30s), get the colony counting with the same method before as in (Tab. 4).

Table 4 | DH5 $\alpha$ spray viable colony counting for aerosol travel distance in $2 \mathrm{~m}$ and $3 \mathrm{~m}$ under the condition of all the designed UV lamps switched on to verify the UV ultimate method. (Spraying center, a 40W UV germicidal lamp, $1.5 \mathrm{~m}$ height; each sampling point, an $8 \mathrm{~W}$ UV germicidal lamp, $1 \mathrm{~m}$ height, all are double-capped quartz glass.)

colony /plate

\begin{tabular}{lcccc}
\hline The UV lamp is & min after UV lamps on & $0 \mathrm{~m}$ & $2 \mathrm{~m}$ & $3 \mathrm{~m}$ \\
\cline { 2 - 5 } continuously switched on, & 0 & $0,0,0,0,0$ & $0,0,0,0,0$ & $0,0,0,0,0$ \\
people on-site use self- & 20 & $0,0,0,0,0$ & $0,0,0,0,0$ & $0,0,0,0,0$ \\
made UV protections or & 40 & $0,0,0,0,0$ & $0,0,0,0,0$ & $0,0,0,0,0$ \\
commercial products for & 60 & $0,0,0,0,0$ & $0,0,0,0,0$ & $0,0,0,0,0$ \\
protection & & &
\end{tabular}


The result demonstrates that Airborne Infection UV Quarantine ${ }^{\circledR}$ Device or UV ultimate quarantine methods can $100 \%$ stop the infectious agent in the airflow while UV germicidal lamps are switching on. Under the UV radiation condition, the viable traveling distance of even the most challenging small-sized aerosol particles with the viable agents is 0 -meter. The difference between the $30 \mathrm{~min} \mathrm{UV}$ protocol and the UV ultimate method is whether people can go on-site while the UV lamp is switching on. The 30min UV protocol only operates while no person is on-site; therefore, it still offers some traveling opportunities for viral aerosols. Also, it can't prevent the later infectious agents from people after the UV radiation is switched off. The UV ultimate method with people put on Infection UV quarantine device to go on-site never gives seconds for the virial aerosol to travel; it is truly a powerful method for killing infectious agents under almost all public life conditions. The only concern is the safety of UV protection instead of the infectious agent. (Albeit UV radiation has been substantially reported to use for in vivo surgical wound $^{12}$ which means the radiation has already reached human naked skin and deep tissues, it is generally believed that UV radiation can't be shining on naked skin or eyes. Later safety validation is still based on this notion.)

\section{Method to test the safety of the self-made or commercial UV protection}

For the safety concern of diverse UV protection, we can easily test by a portable UV meter. (Shielded by the UV protection and attach the sensor to the shielded wall which faces the user, if the radiation is below the safe threshold, then it is safe. Need to check the whole UV protection area.) The physical life of UV germicidal lamps is generally 8,000 - 16,000 operating hours when the lamp is switched on once per day. The actual life will be less due to the frequent operating. Germicidal UV lamps generally define the UVC $253.7 \mathrm{~nm}$ radiation decay to $70 \%$ as the end of the functional hours. The UV radiation strength is inversely proportional to the square of the distance parameter to the emission source; therefore, UV lamps are generally installed to less than $2.5 \mathrm{~m}$ height, and the measuring of strength uses the 1-meter perpendicular distance from the lamp. (Fig.2a) is a portable UV meter which fits for measuring the radiation performance curve or functional life of a UV lamp, we can see the measuring span for this product is $3999 \mu \mathrm{W} / \mathrm{cm}^{2}$ to $39.99 \mathrm{~mW} / \mathrm{cm}^{2}$, this range is not the best candidate for testing the safety of UV protection. It is better to use a high-resolution product like that in (Fig.2b), we can see the resolution is $0.1 \mu \mathrm{W} / \mathrm{cm}^{2}$; this value has already quite lower than in the sunlight. Besides this type of product, we also can alternatively use a portable Geiger counter as in (Fig.2c) for the purpose. The collected slits of this product shown in (Fig. 2d), open the out case we can see the Geiger tube below the slits in (Fig.2e). For this type of alternative product, they are oversensitive and generally have an environmental radiation background. They are designed to measure the radiation of beta radiation $(\beta)$, gamma radiation $(\gamma)$, and part of the $x$-ray from the environmental materials. We need to switch off the UV lamps and get the environmental read, switch on the UV lamp after the shield to test the self-made protection, any read shifting lower than $10 \%$ will be quite safe. There are many varieties of products similar to (Fig.2a-c) on the market with a publicaccessible-price; they can easily allow people to know whether the UV protections they self-made or purchased are safe for use. With them, the UV quarantine system is easy to make into a quite safer system for public life. 
Figure 2 | Implementation of the "Airflow Inaccessible Distancing" code in an ordinary retailed store (14 $\mathbf{m}^{2}$-storefront), a passenger car by "plastic film system"; and the instrument for measuring the safety of UV protections

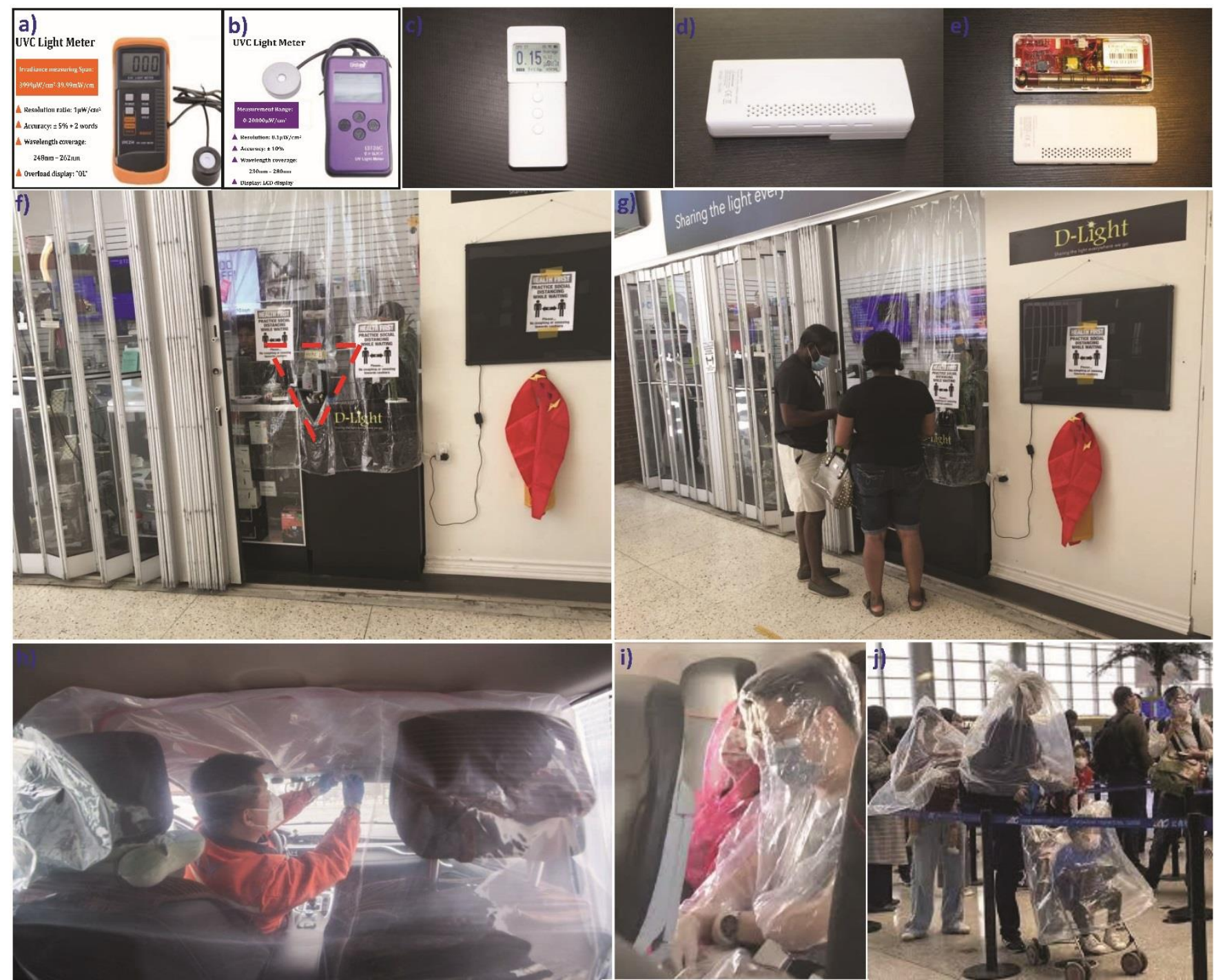

a) a UV 254 meter that fits to detect the functional life of a UV germicidal lamp. b) a UV meter with a resolution of $0.1-\mu \mathrm{W} / \mathrm{cm}^{2}$ which fits for evaluating the safety of a self-made UV protection. c) a portable Geiger Counter. d) the collecting slits of the Geiger counter. e) the Geiger tube below the slits, it claimed to capture and report even one particle that attains this tube. f) a common retailed store with $14 \mathrm{~m}^{2}$ storefront is isolated from outside airflow exchange by a plastic film, only leaves a small hole as inside the red-colored region. $\mathrm{g}$ ) a couple purchased at the store; we can see the hole is in their elbows' height. This method combines with the UV quarantine system can greatly cut down virus spreading. This is a partial separation system with independent respiratory air. h) a passenger car using a plastic film system to separate the drivers with the clients. This is a full separation system with independent respiratory air. Definitely, this plastic quarantine system needs to combine with the UV 30 min protocol for continued usage. i) This way of using plastic film only possesses limited effects in a short period, since it fails to supply separated breath air. j) same with (Fig. 2d), this way of using plastic film still only with limited effect in a short period. The same failure reason with those in (Fig.1a, 1b) due to restricted breath air exchanging. "Airflow inaccessible distancing" code widely adapts to all human public life conditions.

\section{Implementation of the "Airflow Inaccessible Distancing" code by a simple plastic film}


As mentioned, the mask designs in (Fig.1a-b) can't prevent the infection since they allow the airborne viruses to invade into. UV quarantine method is effective since it can actively kill the contagious agents in airflow. Airflow shut systems take a different mechanism; it stops the airborne infectious agents by stopping the airflow, equivalently complies with the "Airflow Inaccessible Distancing" code. (Fig. 2f) shows an ordinary $14 \mathrm{~m}^{2}$-storefront retail store, we can see there is a simple plastic film to quarantine the storefront and only leave a small hole inside the red-colored region on the film for cashier and products exchanging. It is a highly effective physical airflow quarantine system for preventing the infection. There are three employees in the store; they don't use masks since they have a UV radiation box inside the store. The coronavirus must be carried by the airflow for infection; now, the $14 \mathrm{~m}^{2}$ storefront area of air exchange size is restricted to a small hole, the chance of airborne virus invading is greatly diminished. As in (Fig. $\mathbf{2 g}$ ), a couple purchasing at the plastic film window, the hole locates at their elbow height, also greatly reduce the aerosol virus invading. A plastic film is transparent and will not impact their business so much, just every morning a 30 min UV radiation protocol before entering the store, then light up the UV radiation box for healthy employees. (The morning UV disinfection will shine on both sides of the plastic film, albeit it looks transparent. The method of E. coli spraying can verify the process easily. In the spray experiment, add a simple plastic film to stop airflow can effectively stop any sprays from penetrating through the film, then, the "airflow inaccessible distancing" reduced to zero beyond the plastic film. For this store, on the entire $14 \mathrm{~m}^{2}$-storefront, the E. coli sprays can't invade into the room unless directly spray from the hole, and inside the store, ventilation is on the roof which is safe for in-store people to get breath air.) This simple plastic film, combined with the UV radiation box, will be much more effective to prevent the COVID-19 infection than the conventional quarantine system. Most of the retail stores, gas stations, pharmacies, clinic rooms, border customs, bank counters, etc., can use this simple plastic film to shut off airborne infections.

(Fig. 2h) shows a passenger car using a plastic film system to separate the drivers from the clients. The effectiveness of the plastic film system still bases on the "airflow inaccessible" principle. The separation of this car is effective since it offers independent breath air. Definitely, this plastic film quarantine system must combine with a UV 30 min protocol for continued usage; without UV disinfection, plastic film can only use for once. It should be noted that the plastic film usages in (Fig.2i, $\mathbf{2 j}$ ) can only offer limited effect in a short period since they fail to supply separated breath air. The average human respiratory rate is 3060 breaths per minute at birth [24], decreasing to 12-20 breaths per minute in adults [25]. Estimation of the residual volume is average $18.1 \mathrm{ml} / \mathrm{kg}$ for infants [26] or in a proportion of vital capacity for adults 
(0.24 for men and 0.28 for women) [27]. Once people need the breath air, such a way of plastic film usage will fail to issue separate functions since it fails to stop airflow transmission, with the same failure reason with those devices in (Fig.1a, 1b). On an international airplane in which passengers cram inside one enclosed flight cabin for long hours. We have to find some advanced devices which can supply enough sterilized human respiratory air for people.

5. Implementation of the "Airflow Inaccessible Distancing" code by engineering an on-device airflow steering structure to confine all human respiratory air pass it can get sterilization under the UVC radiation maximum performance range via patented Airborne Infection UV Quarantine ${ }^{\circledR}$ Medical Devices. (Canadian Class II medical device ID: 319987 HA\#, 319987 HC\#; HA\# type for adult and HC\# type for children. The common name of which is "UV mask", which means we use $253.7 \mathrm{~nm}$ UVC to sterilize respiratory air before inhale. It takes the UVC sterilization mechanism instead of the "filtering" or "absorption" mechanism like various conventional devices. Note: The UVC in the quarantine device is to sterilize the airflow pass the device instead of disinfecting a conventional mask or device for "reuse".)

The sterilization efficiency of UV germicidal lamps fully relies on the UVC 253.7nm radiation strength. (For some public regions that allow the use of UV lamp induced ozone, the $185 \mathrm{~nm}$ radiation as the ozoneinducing wavelength also extends the effects of the $253.7 \mathrm{~nm}$ UVC radiation of a product.) Regulations of most countries required that the UVC $253.7 \mathrm{~nm}$ radiation strength for sterilization purposes must reach 70 $\mu \mathrm{W} / \mathrm{cm}^{2}$ tests at the $1 \mathrm{~m}$ distance from the device. (Once the $70 \mu \mathrm{W} / \mathrm{cm}^{2}$ strength decays to $70 \%$ to $50 \mu \mathrm{W} / \mathrm{cm}^{2}$, the functional life of a product will be ended. In some of the heavily contaminated regions, the limit has increased to $90 \mu \mathrm{W} / \mathrm{cm}^{2}$, and also the sterilization time must be extended.) The UVC radiation strength of a germicidal lamp follows the inverse-square law which states the strength is inversely proportional to the square of the distance from the source:

$$
U V C \text { radiation intensity }=k \frac{1}{\text { distance }^{2}}
$$

then, $\quad \frac{\text { intesity at } 100 \mathrm{~cm} \text { distance }}{\text { intensity at } 1 \mathrm{~cm} \text { distance }}=\frac{(\text { distance of } 2 \mathrm{~cm})^{2}}{(\text { distance of } 100 \mathrm{~cm})^{2}}=\frac{1}{100^{2}}$

On the market, diverse brands of UV germicidal lamp products generally need at least 36Watt active powers to reach the regulated $70 \mu \mathrm{W} / \mathrm{cm}^{2} \mathrm{UVC}$ radiation strength. So we chose one 36 watt UVC lamp that just meets the limit which tests $91 \mu \mathrm{W} / \mathrm{cm}^{2}$ at 1-meter, one UVC lamp that well over the limit which tests $162 \mu \mathrm{W} / \mathrm{cm}^{2}$ at 1-meter, and also a $500 \mathrm{~W}$ sterilization station for analysis in (Tab. 5). (Generally, UVC germicidal lamps used in hospitals and public regions for sterilization purposes are all under 300W, those over 300W UVC germicidal lamps from a single quartz glass tube are only used for sewage 
treatment. The 500Watt UVC germicidal lamp issued from a single quartz glass tube is the largest active power product on the market we can ever find.) We first test the actual performance of each lamp for sixpoint sterilization distances, then calculate base on a 1-meter result. E.g., the $36 \mathrm{~W}$ lamp test $91 \mu \mathrm{W} / \mathrm{cm}^{2}$ at the 1-meter distance, then we can use the above formula to theoretically calculate the UVC radiation strength at $1 \mathrm{~cm}$ distance from the above equation:

$$
91 \mu \mathrm{W} / \mathrm{cm}^{2} \times \frac{100^{2}}{1^{2}}=910,000 \mu \mathrm{W} / \mathrm{cm}^{2}
$$

Then use the same way for the rest distance points for theoretical calculations as in (Tab. 5). (We can see, the actual test results in (Tab.5) are somewhat different from theoretical calculations, that is due to the inverse-square law only correct for the point sources, and UV lamps are indeed not pointed sources at the short distance. However, such a difference doesn't impact our product analysis and design.) From the result, no matter for a standard regulated UV lamp, a well over limit UV lamp, or even the powerful 500Watt UVC sterilization station, all follow the trend with the increasing of the sterilization distance, the radiation strength decays quickly, albeit some discrepancies away from the inverse-square law.

Table 5 | 253.7nm UVC radiation strength of on-device UVC lamps (319987UVD\#), regulated UVC desktop germicidal lamps (319987UVD\#), and a 500Watt sterilization station

\begin{tabular}{|c|c|c|c|c|c|c|}
\hline Distance of test $(\mathrm{cm})$ & 1 & 2 & 10 & 20 & 50 & 100 \\
\hline U-shape UVC lamp sample 2 & 2013 & 1387 & 189 & 22 & 5.6 & 2 \\
\hline \multicolumn{7}{|l|}{ 2.2W on-device UVC lamp: 319987UVH\# $\left(\mu \mathrm{W} / \mathrm{cm}^{2}\right) *$} \\
\hline U-shape UVC lamp sample 1 & 10660 & 4200 & 400 & 100 & 20 & 8 \\
\hline ring-shape UVC lamp sample 1 & 1884 & 834 & 80 & 27 & 2.3 & 0.7 \\
\hline ring-shape UVC lamp sample 2 & 1735 & 862 & 124 & 20 & 3.5 & 1.4 \\
\hline ring-shape UVC lamp sample 3 & 1521 & 854 & 112 & 33 & 2.6 & 0.8 \\
\hline \multicolumn{7}{|l|}{ regulated UVC lamp $\left(\mu \mathrm{W} / \mathrm{cm}^{2}\right), 36 \mathrm{~W}$, just meet limit } \\
\hline theoretical calculation (based on $91 \mu \mathrm{W} / \mathrm{cm}^{2}$ at $1 \mathrm{~m}$ ) & 910000 & 227500 & 9100 & 2275 & 364 & 91 \\
\hline \multicolumn{7}{|l|}{ regulated UVC lamp $\left(\mu \mathrm{W} / \mathrm{cm}^{2}\right),>38 \mathrm{~W}$, well over limit } \\
\hline actual test result & 34020 & 22150 & 5782 & 2818 & 601 & 162 \\
\hline \multicolumn{7}{|l|}{500 W UVC sterilization station $\left(\mu \mathrm{W} / \mathrm{cm}^{2}\right)$} \\
\hline theoretical calculation (based on $1226 \mu \mathrm{W} / \mathrm{cm}^{2}$ at $1 \mathrm{~m}$ ) & 12260000 & 3065000 & 122600 & 30650 & 4090 & 1226 \\
\hline actual test result & 83450 & 53890 & 17320 & 8600 & 3176 & 1226 \\
\hline
\end{tabular}

* Here don’t perform theoretical calculation for on-device UVC lamp 319987UVH\# 
From the table, if we can make the sterilize distance as close as to that $1 \mathrm{~cm}$, then the airflow sterilization strength of which will be enlarged exponentially. (Fig. 3a) shows such a design; it is technologically called COVID-19 Airborne Infection UV Quarantine® Medical Device (319987HA\#, HC\#) and the common name of which is a UV mask. (Fig. 3b) is the device side view.

Figure 3 | Implementation of the "Airflow Inaccessible Distancing" code by engineering an on-device airflow steering structure to assure sterilization process under the UVC radiation maximum performance range (distance)

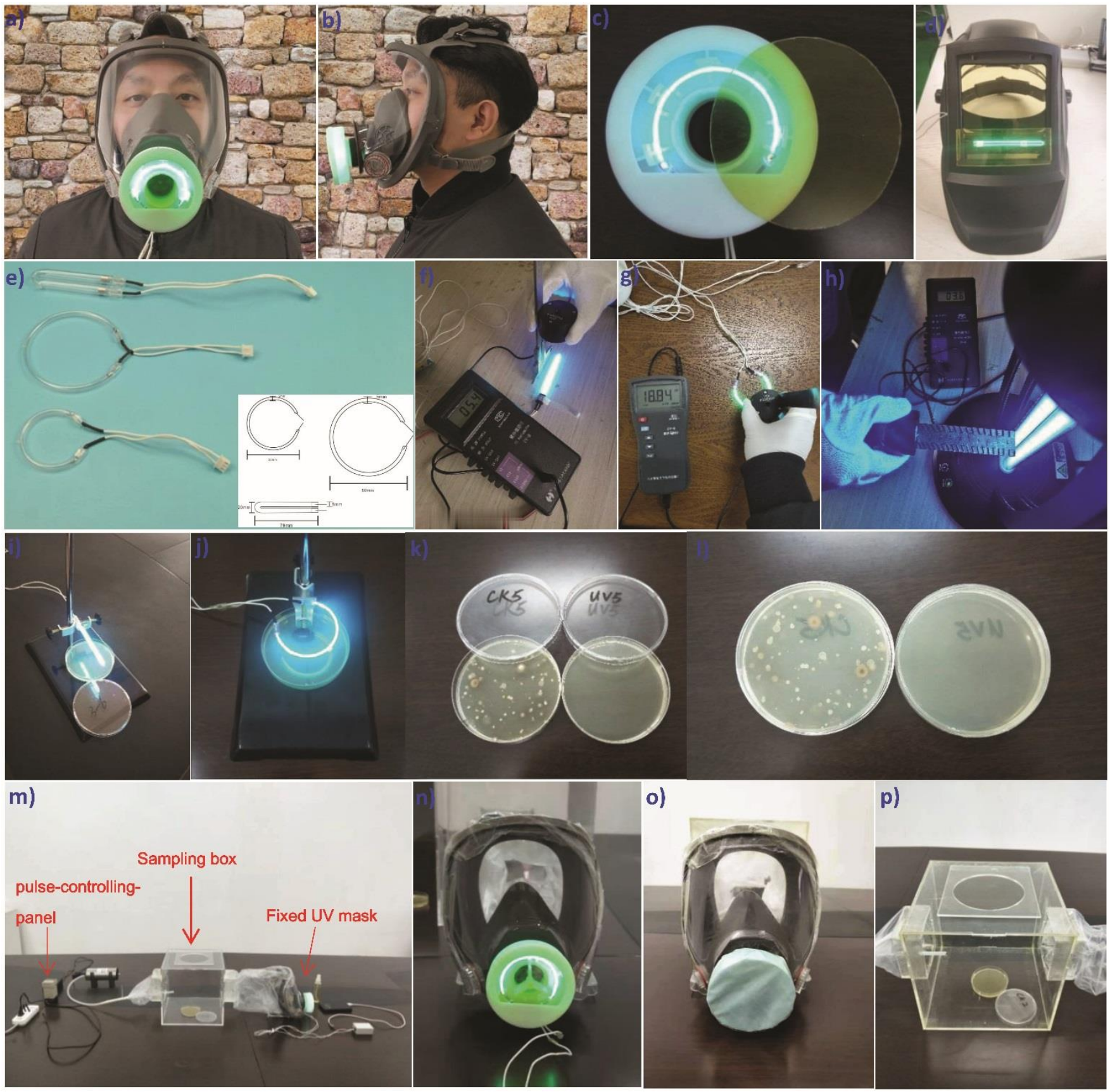


a) an Innoen ${ }^{\circledR}$ COVID-19 airborne infection UV quarantine ${ }^{\circledR}$ device or UV mask ${ }^{\circledR}$ (319987HA\#). In designing, they must follow strict industrial airtight tests to assure that the air leaking $<1 \%$ while fixing on a human face, this limit is quite different from that of the conventional masks which totally neglect the airflow leaking out from the "naked skin contact margin". b) side view of the device. c) the on-device UV germicidal lamp (319987UVH\#) case. There is a UV protective cover on the case to avoid UVC radiation hurt nearby people, and now this UV protective cover is partially opened. This cover seems translucent and we can see the UV lamp under it. However, the materials of it can strongly resist UVC radiation. After passes it, $8000 \mu \mathrm{W} / \mathrm{cm}^{2} \mathrm{UVC}$ radiation will be reduced to less than $8 \mu \mathrm{W} / \mathrm{cm}^{2}$. (Supplementary Movie 2) shows the operation of the UV protective cover of another type of UV mask. d) one type of UV mask (319987HA\#) with a U-shape UVC germicidal lamp (319987UVH\#). e) U-shape and ring-shape on-device UVC germicidal lamps (319987UVH\#). f) test the radiation strength of a U-shaped UVC germicidal lamp. g) test the radiation strength of a ring-shaped UVC germicidal lamp. h) test the radiation strength of a desktop UV germicidal lamp (319987UVD\#). i) petri dish experiment with a U-shape on-device lamp. j) petri dish experiment with a ring-shaped on-device lamp. k) 1) Patri dish experiments of the previous U-shaped and ring-shaped on-device UV lamp at $10 \mathrm{~cm}$ distance. $\mathrm{m}$ ) a system for simulation of human respiratory to validate the performance of UV masks and conventional masks. n) a UV mask fixed on the head part of the system for test, we can see the on-device UV germicidal lamp is on. o) a conventional mask fixed on the head part of the system for test. (We should realize there is no "naked skin contact margin" problem for such a test mask fixing method since we use glue to avoid airflow leaking. However, in a real application for conventional masks, this problem does exist widely due to the "naked skin contact margin" defect of conventional masks.) p) the middle sampling box is made from acrylic materials, we can open the cover and put in Petri dishes, and then seal it into airtight for sampling. Take UVC radiation or EO for sanitation before experiments.

(Fig. 3c) shows the quartz glass UV germicidal lamp (319987UVH\#) shinning inside the case (airflow steering structure) installed on the respiratory region of the device. It is this small engineered structure that steers all the respiratory air of the user to get UVC $253.7 \mathrm{~nm}$ radiation within a $1 \mathrm{~cm}$ distance from the lamp (actual design as short as 6mm). (Fig. 3e) shows U-shaped and ring-shaped on-device UVC germicidal lamps. From (Tab. 5) we can see, the $2 \times 1 \mathrm{~W}$ U-shaped on-device UVC lamps can guarantee over $4000 \mu \mathrm{W} / \mathrm{cm}^{2}$ UVC radiation, and $1 \times 1-2.2 \mathrm{~W}$ U-shaped on-device UVC lamps can guarantee over $8000 \mu \mathrm{W} / \mathrm{cm}^{2} \mathrm{UVC}$ radiation strength, and even $2 \times 1-2.2 \mathrm{~W}$ ring-shaped UVC lamps can guarantee over $3000 \mu \mathrm{W} / \mathrm{cm}^{2} \mathrm{UVC}$ radiation strength to all human respiratory air pass from it. (We have to use 40 folds over-limit UVC radiation strength for sterilization due to human breath is a 6-20L/min moving airflow instead of a static air.)

In contrast, without the above UVC germicidal lamp airflow steering structure, even if we use the powerful 500Watt sterilization station for room respiratory air sterilization, just on the 1-meter distance, the UVC radiation has been reduced to $1226 \mu \mathrm{W} / \mathrm{cm}^{2}$. In the real world, it is truly challenging to arrange such sterilization stations for every 1-meter distance of the room space; therefore, some corners in the room possibly even can't get the regulated lower limit of $70 \mu \mathrm{W} / \mathrm{cm}^{2} \mathrm{UVC}$ radiation. Such a lower than limit sterilized corner is a risk for infection. The patented UV mask, only with $2 \times 1 \mathrm{w}$ or a $1 \times 2 \mathrm{w}$ on-device UV germicidal lamps, then easily defeats the powerful UVC sterilization equipment on the markets to supply infection-free human respiratory air sterilized by over $3000 \mu \mathrm{W} / \mathrm{cm}^{2}$ persistent UVC radiation for the users. Under such an over $3000 \mu \mathrm{W} / \mathrm{cm}^{2}$ UVC radiation, no virus can survive for over 0.1 seconds. This means 
the airflow confine device does possess the ever-known highest human respiratory air sterilization efficiency. (In the literature, the eradication time of bacteria at $10 \mathrm{~cm}$ distance for tested specimens is lower than 15 seconds with $0.318 \mathrm{~J} / \mathrm{s} / \mathrm{m}^{2}$ radiation strength [23]. The data of $0.318 \mathrm{~J} / \mathrm{s} / \mathrm{m}^{2}$ in the paper equal to $31.8 \mu \mathrm{W} / \mathrm{cm}^{2}$, a UV germicidal lamp which can issue $31.8 \mu \mathrm{W} / \mathrm{cm}^{2}$ at $10 \mathrm{~cm}$ even fails the officially regulated radiation standard of $70 \mu \mathrm{W} / \mathrm{cm}^{2}$ to be tested at $1-$ meter. However, even such a poor quality UVC lamp still can eradicate E. coli, etc. within 15 seconds. The official standard of $70 \mu \mathrm{W} / \mathrm{cm}^{2}$ tests at the 1-meter distance has been validated for decades in many countries by diverse methods. Base on this standard, we can understand the powerful sterilization effects from UVC radiation strength over $3000 \mu$ $\mathrm{W} / \mathrm{cm}^{2}$ for infected human respiratory air. ) Most importantly, these in vitro devices don't induce any side effects like those of in vivo interventions such as drugs or vaccines, not only can $100 \%$ eradicate SARSCoV-2 viruses but also can equivalently eliminate other known airborne infectious agents, such as ebola, or even common flu, etc., with every known highest efficiency to stop spreading among people.

6. The device or system for on-site validation of the performance of $\mathbf{U V}$ masks and conventional masks based on the simulation of human lung respiratory pulse and plate sampling

(Fig. 3m) shows a system for evaluating the performance of UV masks. It is composed of three parts: a head which can fix a mask for a test (Fig.3n), a middle sampling box (Fig.3p) which can put in Petri dishes for sampling, and a pump with a pulse-controlling-panel at the back. The human respiratory rate is 30-60 breaths/min at birth [24], 12-20 breaths/min in adults [25], and respiratory volume is around 6-8 liter/min. The pulse-controlling-panel part is used for setting up these parameters. (Supple. Movie 3) shows how this system is running, we even can see the simulation rhythm from the plastic bags. We use it to check whether such respiratory parameters will impact the viable colony counting from E. coli spray simulation as in (Tab. 6). We sterilize the system with UVC or ethylene oxide before each experiment, prepare $10^{6}$ E.coli suspensions to spray at the same level of mask inlet from $1 \mathrm{~m}$ distance pointed to it but not allow visual droplets attain the inlet (The horizontal distance which the visual spray fog can attain is less than $60 \mathrm{~cm}$. This way can assure only aerosols instead of droplets flow into the sampling system.). The spray is manually doing for 60 pulse/min pointed to the air inlet and agrees with the pump pulse. While sampling, put 4 plates in the middle sampling box and seal for the experiment. At the 5 min time point, close the cover, pick plates and seal with parafilm, incubate plates at $37^{\circ} \mathrm{C}$ for 48 hours, counting. 
Table 6 | the impact of human respiratory rhythm to infection rate simulated by E. coli spray viable colony counting via the device for validation conventional masks and UV masks (Fig.3m)

\begin{tabular}{|c|c|c|c|c|c|c|c|}
\hline \multirow[b]{2}{*}{ device parameters } & \multirow{2}{*}{$\begin{array}{c}\begin{array}{c}\text { capturing } \\
\text { time }\end{array} \\
(\mathrm{min})\end{array}$} & \multicolumn{6}{|c|}{ colony counting (colony/plate) } \\
\hline & & group 1 & Ave & STD & group 2 & Ave & STD \\
\hline No pulse, $6 \mathrm{~L} / \mathrm{min}$ & 5 & $13,9,11,7$ & 10.0 & 2.58 & $9,14,13,17$ & 13.25 & 3.30 \\
\hline No pulse, $8 \mathrm{~L} / \mathrm{min}$ & 5 & $15,12,18,9$ & 13.5 & 3.87 & $12,23,11,8$ & 14.25 & 3.59 \\
\hline 12 pulse $/ \mathrm{min}, 6 \mathrm{~L} / \mathrm{min}$ & 5 & $85,76,81,64$ & 76.5 & 9.11 & $76,92,88,81$ & 84.25 & 7.14 \\
\hline 12 pulse $/ \mathrm{min}, 8 \mathrm{~L} / \mathrm{min}$ & 5 & $101,84,91,98$ & 93.5 & 7.59 & $99,111,106,93$ & 102.3 & 7.89 \\
\hline 30 pulse $/ \mathrm{min}, 6 \mathrm{~L} / \mathrm{min}$ & 5 & $96,108,120,95$ & 104.8 & 11.75 & $98,100,91,107$ & 99.0 & 6.58 \\
\hline 30 pulse $/ \mathrm{min}, 8 \mathrm{~L} / \mathrm{min}$ & 5 & $115,120,127,103$ & 116.3 & 10.11 & $108,111,135,124$ & 119.5 & 12.45 \\
\hline
\end{tabular}

From (Tab. 6) we can see, for the same volume of airflow, the viable colony counting for the pulse set up with human respiratory rate is significantly higher than the stable airflow for the system, around 7-8 folds for 12 breaths/min, and 8-9 folds for 30 breaths/min. Here we don't discern in each respiratory cycle, which time point is the competence state for the invading agents to grow. For example, a 12 breaths/min or 30 breaths/min means a 5-second or 2 second period; we yet don't know which 0.1 second is the infection competence stage peak for these 2 or 5 seconds. However, we do know that human breath rhyme does greatly impact E. coli viable colony counting.

In a pandemic world, people try to know how much concentration of SAR-VOR-2 viruses in the aerosol will induce infection. It is so challenging that till now no exact report is available; there is only one report claim $10^{1}-10^{3}$ viable viral particles [28] from estimation. Our E.coli spray simulation system has never used the SAR-VOR-2 viruses or synthesis DNA segments spray, however, E.coli viable colony counting does reveal the stronger correlation between human respiratory rhythm and viral infection concentration, which sustain the above lower limit of the estimation. Suppose human respiration is stable airflow of 6$8 \mathrm{~L} / \mathrm{min}$ and will inhale $\mathrm{X}$ concentration of viral particles. Once these stable airflows change to human real respiration such as $12 \mathrm{pulse} / \mathrm{min}$ or $30 \mathrm{pulse} / \mathrm{min}$, the inhaled concentration of viruses will be increased to 7-9 X. We could understand the reason that human respiratory rhymes increase viable colony counting is due to the exponential increase of whirlpools in the airflow. And the airflow whirlpool holding efficiency is strongly related to the specific surface area. The larger the specific surface area of a system, the more viable colony counting it will hold under a pulse airflow condition. In our sampling box, we only put in 4 Petri dishes in the middle (totally can put 9), which means the specific surface area is very lower. In a pair lobe of human lungs, pulmonary alveoli offer the huge specific surface areas which exponentially larger than our sampling Petri dishes, thus human lungs will hold exponentially more inhale infectious agents 
than our sampling system under rhyme condition, or $>>7-9 X$ which we acquired in (Tab.6). (We should carefully note, this 7-9X doesn't mean the total number of infectious agents inside the airflow get enlarged for so many folds, the total number of infectious agents inside airflow possibly not change so much; what makes the viable colony counting change so dramatically is the simulated human respiratory rhyme or the physical whirlpools inside the airflow.) This fact means, even a very lower concentration coronavirus, possibly one virus particle, will be held inside human pulmonary alveoli due to the above human respiratory pulse effect which we simulated in (Tab.6), and the number of the holding viral particles in lungs will be accumulated with time. (For example, suppose a person with each breath only has one viral particle that can break through the immune systems and finally invade the alveoli, in just 30 minutes, hundreds of viral particles will then accumulate there due to the human respiratory rhyme. These hundreds of viral particles can accumulate from different public regions, such as groceries, dining halls, libraries, supermarkets, etc. The SARS-VOR-2 incubation period inside human bodies will be more than three days complicated the situation. Once the additive effect induced viral proliferation process on human tissue is activated, the infection becomes irreversible. This is also the reason why vaccines are physically so challenging to stop aggressive infection: the artificially induced antibodies need to combat the continuously increasing viral particles accumulating from human respiratory rhyme, and the artificially immunized response generally quite specific, lacks the multi-level system reactions as innate immunity responses established from a long term evolution.) For these reasons, it is less likely to get a general viral particle concentration as the COVID-19 infection guideline. This is also the reason why we must use the pulse-controlling-panel in our evaluation system. Our later on-site evaluation of UV masks or conventional masks is then set up to $12 \mathrm{pulse} / \mathrm{min}$ and $30 \mathrm{pulse} / \mathrm{min}, 6-8 \mathrm{~L} / \mathrm{min}$, this will closer to the real world. Otherwise, data acquired under a stable airflow will deviate from the facts too widely.

Table 7 | on-site validation of the performance of UV mask 319987HA\# by E.coli spray simulation

\begin{tabular}{ccccccc}
\hline & \multicolumn{1}{c}{ capturing time } & \multicolumn{5}{c}{ colony counting (colony/plate), } \\
\hline device parameters & $(\mathrm{min})$ & not switch on & Ave & STD & switch on & Ave \\
\hline $12 \mathrm{pulse} / \mathrm{min}, 6 \mathrm{~L} / \mathrm{min}$ & 5 & $92,109,81,73$ & 88.75 & 15.59 & $0,0,0,0$ & 0 \\
$12 \mathrm{pulse} / \mathrm{min}, 8 \mathrm{~L} / \mathrm{min}$ & 5 & $111,87,95,93$ & 96.5 & 10.27 & $0,0,0,0$ & 0 \\
$30 \mathrm{pulse} / \mathrm{min}, 6 \mathrm{~L} / \mathrm{min}$ & 5 & $87,118,123,95$ & 105.75 & 17.46 & $0,0,0,0$ & 0 \\
$30 \mathrm{pulse} / \mathrm{min}, 8 \mathrm{~L} / \mathrm{min}$ & 5 & $135,102,97,115$ & 112.25 & 16.96 & $0,0,0,0$ & 0 \\
*** set up the same with those in Table 6, treatments include on-device UV germicidal lamp switches on and not switch on. For switch on group experiment, the on- \\
device UV germicidal lamp (319987UVH\#) needs to turn on for 5 min before the experiment procedure; this time is for stable on-site UVC lamp radiation.
\end{tabular}

Different from the experiment in (Tab. 4) which plates only get UVC radiation at nearby $70 \mu \mathrm{W} / \mathrm{cm}^{2}$, all the airflow in (Tab.7) passes the test UV mask gets UVC radiation of $4200 \mu \mathrm{W} / \mathrm{cm}^{2}$, E.coli spraying aerosols which is enough to produce around 100 colonies on plate agar have been totally disabled to 
survival on Petri dishes by the on-device UVC germicidal lamp (319987UVH\#). UVC radiation strength of $4200 \mu \mathrm{W} / \mathrm{cm}^{2}$ tests at $1 \mathrm{~cm}$ distance is only a lower radiation sample among our mass production products. Due to the design of the UV mask 319987HA\#, all the airflow sterilization processes are happening on the maximum performance range of the on-device UV germicidal lamp 319987UVH\#. The products meet the patent designing target. We should understand, the human respiratory rhyme will increase virial holding or increase infection rate; however, our UVC germicidal lamp will be more efficient under human respiratory pulse condition than for a stable airflow. The human respiratory pulse significantly enhances the UVC sterilizing efficiency. This is also why we must install UVC germicidal lamp $319987 \mathrm{UVH} \#$ on the mask respiratory region.

Now, the next step for the products should be Phase 3 clinical trials, in which we should understand the role of the placebo group infection rate.

\section{Placebo group infection rates of global Phase 3 clinical trials intended for infectious agents}

Recently, there are some of the vaccine companies begin to claim higher COVID-19 infection prevent rates; however, their phase 3 clinical trials seem to neglect the prerequisite "Placebo group infection rate”. We can first look at one vaccine claimed with 43,538 participants, 162 infections observed in the placebo group versus 8 in the vaccine group for those around 43,000 participants. Then, half of the 43000 as placebo group only get 162 infections, means the placebo group infection rate of this clinical trial is around:

$$
2 \times 162 / 43000=0.75 \%
$$

The placebo group without injecting the vaccine only induces a $0.75 \%$ infection rate. Under such a "background" infection rate, the vaccine gets $90 \%$ effectiveness. And this effectiveness still means among every 100 vaccinated people, there will present 10 infected people. Another $94.5 \%$ effectiveness claimed has a similar problem, with more than 30000 US participants, finally, 95 cases infection of which 90 cases were observed in the placebo group versus 5 in the vaccine group. Then we can calculate the placebo group infection rate as the following:

$$
2 \times 90 / 30000=0.60 \%
$$

The $94.5 \%$ effectiveness rate is only based on a $0.60 \%$ placebo group infection rate; therefore, this vaccine still only qualifies to use in an environment with not more than $0.60 \%$ "background" infection rate. And even under such a lower viral concentration background, there will still 5 infected people among every 100 vaccinated individuals. For those public regions with any infection rate higher than these trial 
rates, the effectiveness of their vaccines is totally unknown. As is known, the placebo infection rates of most public regions are indisputably higher than the trial set up. E.g. A bus with 30 people inside it, if one person among them infected, then the placebo infection rate will be $3.3 \%$. In a supermarket with 50 people, if 3 persons of them get infected, then the placebo infection rate will be $6 \%$. For a family with 3 members and one gets infected, then the placebo infection rate will be $33.3 \%$. These are only static placebo infection rates. We should realize that in the real world we have to confront a dynamic placebo infection rate. In a movie theater with 3000 audiences, if there is an infected person inside it, two neighboring audiences have to resist a $33 \%$ placebo infection rate, if the vaccine fails to help them resist this level of background infection rate, these two audiences will become newly infected people and release equivalent concentration of coronaviruses within two hours. (For this reason, it is impossible to claim that the placebo infection rate of $1 / 3000$ audience gets COVID-19 hitting is $0.0000033 \%$ in that movie theater, such kind of dynamic placebo infection rate is much more aggressive than a static placebo infection rate.) An international airline evidently with none of confirmed COVID-19 at the departure, while it landed after 14 hours of travel, 7 people have confirmed of infection. From this routine scene, we could understand that $33.3 \%$ placebo infection rate is the minimum requirement for any vaccine intended for infectious agents. (Also, for the results of E. coli spray simulation in (Tab.6), we still can get a similar conclusion. Human respiratory rhyme exponentially increases the inhale viable viruses; therefore, we must prepare an intervention method with a higher enough placebo infection rate.) The abovementioned two vaccines or other phase 3 clinical trials must demonstrate that their vaccines or drugs can offer at least $33 \%$ placebo infection rates before they can go to the market.

To avoid the risk of above lower than $33 \%$ placebo group infection rate vaccine candidates, the COVID19 Airborne Infection UV Quarantine ${ }^{\circledR}$ Devices, or commonly called UV masks, have officially set an artificial environment to assure the placebo group infection rate over $50 \%$, which is acquired by half healthy people and half infected people. (The public needs to see, after taking interventions, such as vaccines, drugs, or medical devices; they won't get any infection in public life even mixing with a higher number of infected people, not just an "effectiveness value" which detached from a real placebo group infection rate scene. Simply speaking, they only want an intervention that can guarantee while one person of a three-member-family gets infected, the other two members still safe under such an intervention method. This is what a $33 \%$ placebo infection rate meant to them. Some of them even need to see a statement sentence on a Letter of Guarantee or similar documents from the product manufacturer.) 
Supplementary Doc 1 is the phase III clinical protocol for COVID-19 Airborne Infection UV Quarantine ${ }^{\circledR}$ Devices, different placebo infection rates such as a 3-day-50 \%, a 3-day-90\%, and a 3day-99\%, etc., have been set up. An over $50 \%$ placebo infection rate is implementing into every unit of our products and the trial report will publish soon. Most importantly, these placebo group infection rates are acquired by already infected people under the pandemic period instead of experimentally infecting the healthy people, this experimental method complies quite well with the clinical trial ethics. Supplementary Doc 2 is the Letter of Guarantee of these series of medical products, which have included the placebo group infection rates as the background. We believe a $33 \%$ placebo infection rate is the minimum requirement for all Phase 3 clinical trials for all medical devices, vaccines, or drugs intended for aggressive infectious agents such as COVID-19. And base on this $33 \%$ placebo infection rate, the intervention method should try to reach over $99 \%$ prevention rate, a $90 \%$ rate which means there are 10 people will get infection among 100 vaccinated people is indeed too lower since these $10 \%$ infected people will continuously become infectious sources, thus makes the required time for cleaning of viruses among a population becomes extraordinarily longer.

\section{Method Summary}

DH5 $\alpha$ suspension is prepared from the secondary inoculation exponential growth stage culture with LB broth in a $250 \mathrm{ml}$ sterilized flask on a $37 \pm 1^{\circ} \mathrm{C}$ rotary shaker. LB agar plates, the spray bottle, and quartz glass UV germicidal lamps are commercial products. Portable UVC light meters and the Geiger-Müller counter are purchased online. Plastic films can be any commercial product from the markets which can bear persistent UV radiation and will not change transparency. COVID-19 Airborne Infection UV Quarantine® Medical Devices (319987HA\#, HB\#), on-device UV germicidal lamps (319987 UVH\#) are from the manufactories. The test box for UV masks and conventional masks is designed and ordered from a hand workshop with acrylic materials. The air pump and the pulse control panel are commercial products.

\section{Data Availability Statements}

The datasets generated during and/or analyzed during the current study are available in the Dryad Digital Repository. 


\section{Acknowledgments}

Thanks to a food manufactory (16,500 sq ft) located in Mississauga, Canada, and a retailed store (260 sq $\mathrm{ft}$ ) located in Brampton, Canada, for their hard work during the SARS-CoV-2 pandemic period, for the implementations of the new "Airflow Inaccessible Distancing” code by plastic film method plus UV quarantine system in their facilities.

\section{Authors' contributions}

JLX, ZXW: Conceptualization, Data Curation, Formal Analysis, Investigation, project administration, supervision, validation, visualization, draft; YL, CL, XHC, CY, WYL, QZG, Data Curation, validation, visualization, draft; YYL: Conceptualization, Data Curation, Formal Analysis, Investigation, project administration, supervision, validation, visualization, draft; YYL: Conceptualization, Data Curation, Formal Analysis, Investigation, Methodology, Project administration, supervision, validation, visualization, Wrote and Edit the whole manuscript, Quality documents and Letter of Guarantee.

\section{Financial Disclosure}

The authors received no specific funding for this work.

Innoen ${ }^{\circledR}$ provided salaries for authors JX, CL, CY. Hubei Jucro Electric Co., Ltd. provided salaries for authors ZW, WL, QG. Wuhan Torch Optoelectronic Technology Co., Ltd provided a salary for author YL. These funders provided support in the form of salaries for authors but did not have any additional role in the study design, data collection and analysis, decision to publish, or preparation of the manuscript. The specific roles of these authors are articulated in the 'author contributions' section.

\section{Competing financial interests}

Innoen ${ }^{\circledR}$ provided salaries for authors JX, CL, CY. Hubei Jucro Electric Co., Ltd. provided salaries for authors ZW, WL, QG. Wuhan Torch Optoelectronic Technology Co., Ltd provided a salary for author YL. This work is related to a patent (USA, PCT patent 17067384, Airflow UV Quarantine Method and 
Airborne Infection UV Quarantine Device, Yi Yu Lai). This does not alter our adherence to PLOS ONE policies on sharing data and materials.

\section{Supporting Information}

S1 Movie. E.coli spray over a UV radiation box S2 Movie. UV protective cover on a UV mask

S3 Movie. the device for simulation of human respiratory with pulses to validate the performance of UV masks and conventional masks

S4 Document. PHASE 3 CLINICAL TRIAL PROTOCOL

S5 Document. Letter of Guarantee

\section{References}

1. Ali, S.T. et al. Serial interval of SARS-CoV-2 was shortened over time by nonpharmaceutical interventions. Science 10.1126/science.abc9004 (2020).

2. Aiello, A. et al. Immunosenescence and Its Hallmarks: How to Oppose Aging Strategically? A Review of Potential Options for Therapeutic Intervention. Frontiers in Immunology. 10,1-19 (2019). Doi:10.3389/fimmu.2019.02247

3. Driggin, E. et al. Cardiovascular Considerations for Patients, Health Care Workers, and Health Systems During the Coronavirus Disease 2019 (COVID-19) Pandemic. J Am Coll Cardiol.75(18),2352-2371(2020). doi: 10.1016/j.jacc.2020.03.031

4. Cummings, M.J. et al. Epidemiology, clinical course, and outcomes of critically ill adults with COVID-19 in New York City: a prospective cohort study. The Lancet. 395, 1763-1770(2020) doi:10.1016/S0140-6736(20)31189-2

5. Batlle, D. et al. Acute Kidney Injury in COVID-19: Emerging Evidence of a Distinct Pathophysiology. JASN. 31(7), 1380-1383(2020). DOI: 10.1681/ASN.2020040419

6. Helms, J. et al. Neurologic Features in Severe SARS-CoV-2 Infection. $N$ Engl J Med. 382,22682270(2020) DOI: 10.1056/NEJMc2008597

7. Moriguchi, T. et al. A first case of meningitis/encephalitis associated with SARS-Coronavirus-2. International Journal of Infectious Diseases. 94,55-58(2020). DOI:10.1016/j.ijid.2020.03.062 
8. Cui, S., Chen, S., Li, X., Liu, S., Wang, F. Prevalence of venous thromboembolism in patients with severe novel coronavirus pneumonia. J Thromb Haemost.18,1421-1424(2020). DOI: $10.1111 /$ jth. 14830

9. Tang, N., Li, D., Wang, X., Sun, Z. Abnormal coagulation parameters are associated with poor prognosis in patients with novel coronavirus pneumonia. J Thromb Haemost.18(4),844-847(2020). doi: $10.1111 /$ jth.14768.

10. Lippi, G., Favaloro, E.J. D-dimer is Associated with Severity of Coronavirus Disease 2019: A Pooled Analysis. Thromb Haemost. 120(5), 876-878 (2020). doi: 10.1055/s-0040-1709650.

11. Teuwen, L-A., Geldhof, V., Pasut, A., Carmeliet, P. COVID-19: the vasculature unleashed. Nat Rev Immunol.20(7),389-391(2020). doi: 10.1038/s41577-020-0343-0.

12. Klok, F.A. et al. Incidence of thrombotic complications in critically ill ICU patients with COVID-19. Thrombosis Research. 191,145-147(2020).

13. Dolhnikoff, M. et al. Pathological evidence of pulmonary thrombotic phenomena in severe COVID19. J Thromb Haemost. 18(6),1517-1519(2020). doi: 10.1111/jth.14844.

14. Lai, Y. Y. Quarantine of UV-Shine Itself Instead of Quarantine Infectious Agent as a Physical Control Method is the only Ever-Known Effective Method for Stopping the Spreading Potential of 2019-nCoV in China and Later Possibly Disseminate to the Global. IJSR, 9(3), 27-28 (2020). DOI: $10.21275 /$ SR20228084927

\section{Saturation Secondary Infection curve:}

http://www.innoen.org/index.php?m=collective $\& \mathrm{c}=$ collective $\& \mathrm{a}=\operatorname{article} \& \mathrm{~b}=$ show $\& \mathrm{iid}=\mathrm{d} 3 \mathrm{zq} 7 \mathrm{c} 0 \mathrm{~d} 74$ g8g8sgs4ww8w4sg1601576042405414004283

16. Downes, A., Blunt, T. P. On the Influence of Light upon Protoplasm. Proceedings of the Royal Society of London. 28 (190-195), 199-212(1878). doi:10.1098/rspl.1878.0109.

17. "The Nobel Prize in Physiology or Medicine 1903". Nobelprize.org. The Nobel Foundation. Retrieved 2006-09-09.

18. Morawska, L., Cao, J. Airborne transmission of SARS-CoV-2: The world should face the reality. Environment International. 139, (2020) 105730. doi:10.1016/j.envint.2020.105730.

19. Pollitt, G. et al. COVID-19 vulnerability: the potential impact of genetic susceptibility and airborne transmission. Human Genomics. 14:17,1-7(2020). doi: 10.1186/s40246-020-00267-3. 
20. Jayaweera,M., Perera, H., Gunawardana, B., Manatunge, J. Transmission of COVID-19 virus by droplets and aerosols: A critical review on the unresolved dichotomy. Environment Research 188, (2020) 109819. doi: 10.1016/j.envres.2020.109819.

21. Setti, L. et al. Airborne transmission route of COVID-19: Why 2 meters/6 feet of inter-personal distance could not be enough. Int. J. Environ. Res. Public Health 2020, 17, 2932. doi:10.3390/ ijerph17082932

22. Correia,G., Rodrigues,L., Gameiro da Silvac, M., Gonçalves, T. Airborne route and bad use of ventilation systems as non-negligible factors in SARS-CoV-2 transmission.

23. Rao, B.K., Kumar, P., Rao, S., Gurung, B. Bactericidal Effect of Ultraviolet C (UVC), Direct and Filtered Through Transparent Plastic, on Gram-positive Cocci: An In Vitro Study. Ostomy Wound Management. 57(7), 46-52(2011).

24. DeBoer, S. L. Emergency Newborn Care. Trafford Publishing. p. 30. ISBN 978-1-4120-3089-2. (4 November 2004).

25. Lindh, W. Q. Et al. Comprehensive Medical Assisting: Administrative and Clinical Competencies. Cengage Learning. p. 573. ISBN 978-1-4354-1914-8. (9 March 2009)

26. Morris, M. G. Comprehensive integrated spirometry using raised volume passive and forced expirations and multiple-breath nitrogen washout in infants. Respiratory Physiology \& Neurobiology. 170(2), 123-140(2010). doi:10.1016/j.resp.2009.10.010.

27. Wilmore, J. H. The use of actual predicted and constant residual volumes in the assessment of body composition by underwater weighing. Med Sci Sports. 1(2), 87-90(1969). doi:10.1249/00005768196906000-00006.

28. Popa, A. et al. Genomic epidemiology of superspreading events in Austria reveals mutational dynamics and transmission properties of SARS-CoV-2. Sci. Transl. Med. 10.1126/scitranslmed. abe2555 (2020). 